\title{
TRAITEMENT ET STOCKAGE DES DÉCHETS RADIOACTIFS
}

\section{Introduction}

\author{
par André GAUVENET
}

Délégué Central Sécurité du C.E.A.

II n'est guère de problème technique lié à l'énergie nucléaire qui soit actuellement plus controversé que celui des déchets radioactifs.

Il faut rappeler cependant les grands principes retenus pour la gestion des déchets radioactifs dans l'industrie nucléaire, aussi bien d'ailleurs que dans l'utilisation, devenue courante, des sources radioactives.

Les rejets d'effluents sont limités à peu de choses et l'on s'accorde désormais - y compris chez les opposants pour considérer que le cycle de l'énergie nucléaire, dans son fonctionnement normal, est très peu polluant. Dans l'avenir, cette situation peut et doit rester satisfaisante, grâce à la mise au point, qui se parfait actuellement, de moyens efficaces de dépollution agissant sur les produits gazeux de période moyenne qui, aujourd'hui inoffensifs, pourraient poser des problèmes à terme.

Qui dit énergie nucléaire dit, d'ailleurs, dépollution aisée grâce à l'ampleur des recherches et au volume modeste des produits en cause. C'est là un avantage certain, et non négligeable, de la concentration de l'énergie dans la matière fissile.

La faiblesse des rejets a pour corrélatif la conservation nécessaire, dans des sites choisis, de déchets plus ou moins concentrés. II faut noter que l'énergie nucléaire pourrait sans aucun doute disperser sans dommages pour l'environnement davantage de produits. Elle se refuse à le faire et cette tendance s'affirmera plus encore dans l'avenir.

Les déchets comprennent deux grandes catégories:
- ceux qui sont peu actifs et présentent par conséquent peu de risques pour l'environnement ;

- ceux qui sont très actifs et qui de ce fait sont dangereux.

Ces derniers sont d'ailleurs très peu volumineux, ce qui compense partiellement leur risque, en facilitant la recherche des sites de stockage.

Dans les deux cas, il faut gérer différemment les produits à faible durée de vie et ceux qui, au contraire, ont de longues périodes.

La politique nucléaire de stockage des déchets utilise des dispositions très sûres pour le court et le moyen terme. Les sites sont choisis après une étude écologique sérieuse et selon une pror cédure rigoureuse. Les techniques de stockage sont particulièrement éprouvées.

Pour le moyen terme, la décision a été prise de vitrifier les déchets de très haute activité, ce qui leur donne une forme particulièrement commode en vue d'un stockage de longue durée (quelques centaines d'années).

Les produits de très longue durée de vie posent certes le problème des nuisances qu'une génération - la nôtre a le droit de léguer à ses successeurs. Mais c'est un problème plus théorique que réel dans la mesure où la radiotoxicité de ces résidus est au plus du même ordre de grandeur que la toxicité des minerais d'uranium qui les ont engendrés. A long terme, donc, le globe ne sera pas plus "radioactif " qu'il ne l'est actuellement. C'est d'ailleurs ce que l'on a constaté à Oklo, au Gabon, où plusieurs réacteurs nucléaires se sont constitués naturellement dans un gisement d'uranium. Après une longue période de fonctionnement, les déchets radioactifs sont restés sur place et ils ont aujourd'hui disparu.

Pour le très long terme, diverses méthodes sont envisagées pour que "l'effet Oklo » de conservation sur place des déchets se produise dans des conditions contrôlées, et sans surveillance. Un dossier déjà très étoffé a été constitué, en même temps que l'on envisage et que l'on étudie la séparation des produits à longue durée de vie et leur éventuelle transmutation vers des produits de durée de vie plus courte.

Pour l'instant, il n'est nullement nécessaire de mettre en service de tels stockages, sauf à titre expérimental et de manière réversible. Nous disposons de beaucoup de temps pour perfectionner nos méthodes et les vérifier.

En fin de compte, il ne faut pas perdre de vue qu'un déchet radioactif est souvent plus facile à traiter et à stocker qu'un déchet industriel. A la différence de ce dernier, le déchet radioactif décroît et disparaît plus ou moins rapidement. La majorité des résidus est d'ailleurs de courte durée de vie.

Pour nous résumer: les difficultés que présentent les déchets sont abordées très sérieusement par les responsables ; les stockages actuels présentent de bonnes garanties et les solutions futures, à très long terme, sont activement étudiées.

Les articles qui vont suivre présentent certains aspects essentiels de cette importante question.

A. G. 